\title{
Avaliação de Múltiplas Visualizações do Modelo do Estudante no Domínio de Programação
}

\author{
Priscylla Silva $^{1,2}$, Igor Santana ${ }^{1}$, José Raniel Correia ${ }^{1}$ \\ ${ }^{1}$ Instituto Federal de Alagoas (IFAL) - Campus Rio Largo - Rio Largo, AL - Brasil \\ ${ }^{2}$ Universidade Federal de Campina Grande (UFCG) - Campina Grande, PB - Brasil \\ priscylla.sousa@ifal.edu.br, \{igoricml,ranielcorreia\}@gmail.com
}

\begin{abstract}
This paper presents a set of eight student model visualizations created for an online educational system that aim is teaching programming for beginners. A questionnaire was applied with 16 programming students to check the quality of the visualizations. The visualizations most simpler have performed better, even providing the more restricted set of information.
\end{abstract}

Resumo. Este artigo apresenta um conjunto de oito visualizações para o modelo do estudante criadas para um sistema educacional online voltado ao ensino de programação para iniciantes. Um questionário foi aplicado com 16 estudantes de programação para verificar a qualidade das visualizações. As visualizações mais simples obtiveram melhores resultados, mesmo forcendo um conjunto de informações mais restrito.

\section{Introdução}

Sistemas educacionais computadorizados armazenam informações referentes as interações dos estudantes com o sistema e as soluções de atividades submetidas por eles. Alguns desses sistemas, como no caso dos Sistemas Tutores Inteligentes, utilizam essas informações para a construção do chamado modelo do estudante, esse modelo é uma representação do estado atual do conhecimento, habilidades e competências de cada estudante individualmente [Bull et al. 2016]. Alguns trabalhos na literatura propõem a abertura desses modelos para que os estudantes possam inspecionar seu conteúdo e verificar a opinião do sistema sobre eles [Zapata-Rivera and Greer 2004]. A abertura do modelo tem a vantagem de promover a reflexão do estudante, fazendo com que ele monitore o seu aprendizado por meio do acompanhamento da evolução do seu modelo e planeje melhor seus estudos.

Neste trabalho foi realizada a criação de oito visualizações para o modelo do estudante de um sistema educacional voltado ao ensino de programação. O modelo do sistema utiliza redes bayesianas para modelar o conhecimento do estudante [Zapata-Rivera and Greer 2004], sempre que o estudante realiza uma nova submissão de exercício no sistema, sua solução é avaliada e, em seguida, o resultado dessa avaliação é utilizado para atualizar o modelo do estudante.

\section{Visualizações do Modelo do Estudante}

A Figura 1 apresenta as visualizações criadas para o modelo do estudante. Elas foram construídas baseadas em uma revisão da literatura sobre o tema [Bull et al. 2016] e podem ser classificadas de acordo com três categorias: visão estrutural, visão de nível de 
VII Congresso Brasileiro de Informática na Educação (CBIE 2018)

Anais do XXIX Simpósio Brasileiro de Informática na Educação (SBIE 2018)

conhecimento e visão social. As visualizações 1, 2 e 3 (ver Figura 1) possuem um foco na visão social e de nível de conhecimento, pois mostram uma comparação do nível de conhecimento do estudante (cor rosa) com o da turma (cor azul). As visualizações $4 \mathrm{e}$ 5 possuem uma visão de nível de conhecimento e estrutural, pois em ambos os casos os tópicos do domínio de programação são organizados em uma estrutura em árvore e cada nó da árvore possui uma representação do nível de conhecimento do estudante, gauges no caso da visualização 4 e emoticons no caso da 5. As visualizações 6, 7 e 8 possuem apenas a visão de nível de conhecimento. A 6 apresenta estrelas indicando o nível de conhecimento do aluno para cada tópico. A visualização 7 exibe os tópicos de programação em uma tabela, cada coluna indica um nível e um checkpoint é marcado sinalizando o nível do estudante no tópico. Por fim, a visualização 8 mostra um mapa de calor, também conhecido como TreeMap, onde os tópicos são regiões no mapa e o nível de conhecimento é indicado pela cor de cada região.



Figura 1. Exemplo de cada uma das oito visualizações criadas no trabalho.

\section{Avaliação das Visualizações, Resultados e Discussões}

Para a avaliação das visualizações foi aplicado um questionário com 16 estudantes de programação. O questionário foi composto por 32 perguntas, onde cada uma das 8 visualizações estava associada a 3 perguntas. Os estudantes foram instruídos de que o questionário conteria um conjunto de visualizações relativas ao conhecimento de um estudante em programação, onde cada visualização tinha por objetivo mostrar o nível de conhecimento deste estudante em tópicos específicos do domínio de programação. As visualizações contidas no questionário foram extraídas de um modelo do estudante gerado de forma aleatória. As perguntas contidas no questionários relativas a cada visualização 
VII Congresso Brasileiro de Informática na Educação (CBIE 2018)

Anais do XXIX Simpósio Brasileiro de Informática na Educação (SBIE 2018)

foram: 1. Considerando a visualização apresentada, na sua opinião, qual o tópico de programação o estudante mais domina? 2. Considerando a visualização apresentada, na sua opinião, qual o tópico de programação o estudante menos domina? 3. Considerando a visualização apresentada, forneça uma nota de zero a dez para a qualidade da visualização (considere a facilidade que você teve em identificar os tópicos pedidos nas perguntas anteriores). Para as perguntas 1 e 2, o estudante poderia escolher apenas uma opção.

Tabela 1. Compilação dos resultados da aplicação do questionário.

\begin{tabular}{|l|c|c|c|c|c||c||c|c|}
\hline Visualizações & $\mathbf{1}$ & $\mathbf{2}$ & $\mathbf{3}$ & $\mathbf{4}$ & $\mathbf{5}$ & $\mathbf{6}$ & $\mathbf{7}$ & $\mathbf{8}$ \\
\hline \hline Acertos pergunta 1 & $81,3 \%$ & $81,3 \%$ & $68,8 \%$ & $93,8 \%$ & $87,5 \%$ & $93,8 \%$ & $93,8 \%$ & $31,3 \%$ \\
\hline \hline Acertos pergunta 2 & $93,8 \%$ & $87,5 \%$ & $62,5 \%$ & $87,5 \%$ & $62,5 \%$ & $93,8 \%$ & $81,3 \%$ & $50,0 \%$ \\
\hline Média da avaliação & 7,75 & 7,37 & 5,12 & 7,06 & 6,94 & 8,75 & 8,25 & 6,56 \\
\hline
\end{tabular}

Os resultados da aplicação do questionário de avaliação das visualizações são apresentados na Tabela 1. Para a pergunta 1, onde foi pedido para o participante identificar na visualização qual o tópico que apresentava melhor desempenho, em 6 das 8 visualizações mais de $80 \%$ dos participantes conseguiu identificar o tópico corretamente. Para a pergunta 2, onde foi pedido para identificar o tópico que apresentava pior desempenho, em 5 das 8 visualizações mais de $80 \%$ dos participantes conseguiu identificar o tópico correto. Em ambos os casos, a visualização que apresentou os melhores resultados foi a visualização 6 , nesta visualização, cada tópico possui 5 estrelas associadas, e a quantidade de estrelas em destaque indica o desempenho do estudante em cada tópico. A visualização que apresentou os resultados mais baixos foi a visualização 8, composta por um TreeMap, que apresenta os tópicos como regiões em um mapa, e a intensidade da cor indica o nível de conhecimento num tópico.

Os resultados das avaliações mostraram que as visualizações mais simples obtiveram melhores resultados, pois os estudantes conseguiram identificar os tópicos do domínio e o nível de conhecimento com maior facilidade. As notas dadas pelos participantes do questionário as visualizações também reforçam essa percepção.

\section{Considerações Finais}

Este trabalhou apresentou um conjunto de oito visualizações para o modelo do estudante de um sistema educacional voltado para o ensino de programação. As visualizações foram avaliadas por meio de um questionários e percebeu-se que as mais simples obtiveram melhores resultados. Como trabalhos futuros, será realizado o confronto do resultado do questionário com o log de uso das visualizações dos participantes no sistema.

\section{Referências}

Bull, S., Ginon, B., Boscolo, C., and Johnson, M. (2016). Introduction of learning visualisations and metacognitive support in a persuadable open learner model. In Proceedings of the Sixth International Conference on Learning Analytics \& Knowledge, LAK '16, pages 30-39, New York, NY, USA. ACM.

Zapata-Rivera, J.-D. and Greer, J. E. (2004). Interacting with inspectable bayesian student models. Int. J. Artif. Intell. Ed., 14(2):127-163. 